\title{
Staying Strong and Healthy: Minimizing Cardiovascular and Metabolic Effects of Androgen Deprivation Therapy: A Study in Transition
}

\author{
Sally L. Maliski, Elisabeth Hicks, Alana Enslein \\ The University of Kansas Medical Center
}

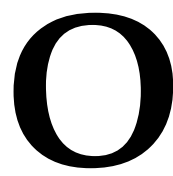

ver the past decade, use of androgen deprivation therapy (ADT) to treat prostate cancer has risen seven-fold, from $9.8 \%$ of patients in $1989-1992$ to $74.6 \%$ in 1991-2001 (1). ADT is used as neoadjuvant and adjuvant treatment with radiation therapy $(\mathrm{RT})$, to treat recurrence following primary treatment with surgery or RT, or when the cancer is in an advanced stage at diagnosis. While ADT has demonstrated survival benefits, it also is associated with increases in metabolic and cardiovascular risks. Men receiving ADT have been shown to develop dyslipidemia, decreased arterial compliance, increased insulin resistance, weight gain with increased visceral fat deposition, decreased bone mineral density, decreased libido and erectile dysfunction, fatigue, cognitive changes and depression (1-12)

Importantly, cardiovascular disease (CVD) is the leading non prostate-related cause of death among men with prostate cancer, accounting for $11-21 \%$ of deaths (13). Men on ADT have a $20 \%$ higher risk of myocardial infarction (MI) and congestive heart failure $(\mathrm{CHF})$ than their counterparts not on ADT (14). Also, data from the Prostate Cancer Outcomes Study (PCOS) (15) indicates that prolonged use of ADT increases the risk for diabetes and depression as well as risk of CVD and related MI and CHF (Penson, personal communication). Further, Latino men are more likely to be diagnosed with later stage disease (16-25) and have been underrepresented in previous studies. Latino men in general have higher rates of metabolic syndrome, diabetes, obesity, and hypertension $(28,34-41)$.

Additionally, we have found that Latino men start treatment for prostate cancer with lower health related quality of life (HRQOL) and that HRQOL declines over time for men on ADT relative to other treatments for prostate cancer (43). However, we have also found that these men are very interested in making changes that will improve their health, as are the wives of the married men (44). Finally, we are finding that Latino men are unable to identify effects of ADT other than hot flashes and sexual dysfunction demonstrating a lack of awareness of the metabolic and cardiovascular effects of ADT. Starting ADT may be a "teachable moment" to intervene with education and support for progressive individualized behavioral changes to minimize cardiovascular and metabolic effects of ADT. Strategies which have been proven to improve risk profiles in other contexts are being used with this population.

Presented here is a description of an $\mathrm{NIH}$-funded randomized controlled trial of intervention to minimize cardiovascular and metabolic risk for Latino men on ADT. Participants will be randomized 
into either an intervention group or an attention control group. Specifically, this study aims to:

- Compare pre- and post-intervention body mass index (BMI), lipid profile, waist/hip circumference and blood glucose levels within and between groups

- Compare pre- and post-intervention HRQOL and depression within and between groups

- Develop and compare explanatory models of nutrition and activity choices between groups

- Evaluate acceptability and benefit of the intervention from participants' perspective

\section{Methods}

We are using a RCT design with the added feature of a constructivist grounded theory (20) component with a subset of 30 participants from the intervention group and 30 from the attention control group.

\section{Recruitment}

We are recruiting 150 Latino men who have initiated ADT within the past 3 months. The study coordinator is working with office staff of urology clinics in a Midwestern city to identify participants who are potentially eligible for the study and obtain permission for study staff to contact the potential participant to explain the study. Inclusion

criteria are:

- Self-identifies as Latino

- Within 3 months of starting ADT

- Diagnosed with prostate cancer

- Able to read and write in English or Spanish

- Not prohibited from exercise by a physician
Men identified by clinic staff and who give permission to be contacted, will receive a call from the bilingual (EnglishSpanish) study coordinator to explain the study, determine eligibility, answer questions and obtain verbal consent. Men will be randomized into the intervention or control group as they enroll. The study coordinator will contact consenting men to schedule the baseline visit at the translational research unit. Men are encouraged to bring their partner or an adult child. Assistance with transportation is provided if needed.

\section{Procedures}

Prior to the baseline visit, the study coordinator will contact all men and instruct them not to eat or drink after midnight before their visit. The coordinator reviews the study, provides direction to the translational research unit and ascertains whether transportation assistance is needed. Men are greeted by the study coordinator upon arrival, introduced to the study nurse, and taken to the unit for their blood draw. Breakfast and beverage are then provided. Next, the study nurse takes the participant's blood pressure, and measures weight, height, waist and hip circumference. The study coordinator and study nurse then administer study instruments including general and disease-specific quality of life measures, a physical activity questionnaire, 24-hour food recall, and depression measure. This concludes baseline measures. The same measures are repeated at six months and 12 months.

Intervention

At the baseline visit, those assigned to the intervention group receive a smartphone. The study cultural liaison instructs men in the use of the phone. All 
materials for the intervention education curriculum have been preloaded onto the phone. Men in the intervention group then receive a physical fitness assessment including upper and lower body strength and $\mathrm{VO} 2$ Max administered by co-I exercise physiologist. The assessment is then used to develop an individualized progress exercise plan and goals. Similarly, a personalized nutrition plan and goals are developed from the nutrition assessment.

Men and their significant other then receive a weekly call for three months from the study nurse and cultural liaison who is bilingual/bicultural utilizing the smartphone. Each week there is an educational component, activity component, nutrition component, and discussion of goal setting relative to activity and nutrition for the following week. The education topic for each week has been preloaded onto the smartphone along with exercise demonstration videos. Supplemental material can be sent to men as needed. Co-Is consult with the study nurse on nutrition and activity for participants as well any medical questions that arise. Additionally, participants are referred to their primary physician when concerns arise. During the next three months, men receive a monthly call from the cultural liaison to monitor, assist, and coach with the nutrition and activity plan established at the end of the three months of weekly calls. Men then return for the 6-month data collection visit. There is no contact between the six-month and 12month data collection visits to ascertain stability of intervention outcomes.

Qualitative Component

A constructivist grounded theory approach is being utilized for this portion of the study to explore differences in decision-making processes related to nutrition and activity between the two groups. A subsample of 30 men from each group will be purposively selected for semistructured interviews focusing on how they made choices about food selections and exercise. Additionally, men in the intervention group will be asked about their experience of the intervention including what was and was not helpful, ease of use of the smartphone, and ability to set, accomplish, and maintain weekly goals.

\section{Analysis}

Analysis will include both quantitative and qualitative techniques. Preliminary analysis will include descriptive statistics for each measure at each time point, assessment of distributional characteristics including outliers, evaluation of missing data, and assessment of reliability. Intervention and control groups will be compared for baseline equivalence on metabolic indicators and HRQOL, as well as other selected factors (e.g. sociodemographics, co-morbidities, medications, primary prostate cancer treatment, acculturation, diet and activity indicators, etc.) using t-tests or chi square tests relevant to the distributional characteristics. The correlation of these possible covariates to the outcome measures will also be examined.

Analyses for the first aim will compare groups in terms of their change in metabolic indicators over time using a mixed effects model for repeated measures, in which group is considered a fixed between-group factor (101, 108). This approach will allow inclusion of covariates determined in preliminary anal- 
yses in order to adjust for baseline individual differences that might impact patterns over time. This will also allow for inclusion of all available data points for each individual, even if the data vector is incomplete due to attrition. Each outcome indicator will be considered a dependent variable in separate models. In these models, the group-by-time interaction term will be examined to determine differences between groups over time. Additional contrasts will examine the shape of the pattern of change over time within each group, specific changes from baseline to 6 months and baseline to 12 months within each group, and the difference between groups at each observation point. Interaction effects will allow further examination of covariate relationships to these differences over time. A similar approach will be used to address the second aim with HRQOL and disease-specific HRQOL as dependent variables.

The third and fourth aims will be met through qualitative analysis of interviews conducted at the 12-month data collection visit using grounded theory techniques (Charmaz 2006). All interviews will be audio-recorded and transcribed verbatim. Transcripts will be entered into Atlas ti.v2 for data management. Initially, the PI will read transcripts in their entirety and proceed to segment-by-segment coding. Related codes will be clustered and developed into categories describing process used by participants to make food and activity choices. The study team will independently code several of the same transcripts and meet to confer and agree on categories. The team will then continue coding and categorizing with periodic meetings to develop full description for each category that includes properties and dimensions and identify relationships among categories. For the intervention group, we will explore categories for explanations of adherence and non-adherence. We will also compare the explanatory frameworks developed for each group relative to nutrition and activity decision making. These explanatory frameworks will be used to contextualize the interpretation of the outcome measures taking into account participants' decision process, context factors which influenced outcomes, perception of importance and relationship of nutrition and activity to HRQOL and health during ADT.

Institutional Transitioning of the Study

At this point, the study is in transition between two major universities. The process is a lengthy and complex one which demands constant communication. An initial step involved identifying the resources and technology at the new institution necessary to conduct the study. This included building the infrastructure with new co-investigators, consultants, as well as hiring a new research manager and research staff. Fortunately, the resources and expertise were available at the new institution and there has been immense support from interdisciplinary teams.

As recruitment is pertinent to study success, it was necessary to be proactive about understanding where the potential participant pool of Latino men starting ADT would be coming from. We found that this pool was much smaller at the new university; however, an African 
American population was more abundant. We thought it would be interesting to explore this underrepresented population as well, and thus, were granted permission by the NIH to expand our recruitment to men of any ethnicity. The new team has been able to identify strategies for recruitment which include working with local urology clinics and community groups for the identification and potential enrollment of participants. During this time, study work at the original institution was being transitioned from recruitment to preparing for the transfer of the study.

Mechanisms for transfer of data collection procedures, documentation, tracking, storage and management had to be developed. This continues to involve collaboration and communication between many different parties, including but not limited to the research manager at the new institution with the study staff at the transferring institution, as well as medical informatics at the new institution to build the framework for data collection and storage. Additionally, study equipment such as smartphones, resistance bands, heart rate monitors, and gift cards for participants had to be moved from one institution to the other.

Finally, there has been ongoing communication with the NIH to meet requirements for relinquishment of the grant by the original institution and acceptance by the new institution. There have been both formal and informal communications to accomplish this. This has included reformulation of the study budget, modification of methods to fit the new site, and obtaining IRB approval at the new institution. During this time, recruitment and study activities have had to be suspended until the grant is officially transferred to the new institution. As there are many moving parts involved in building a new infrastructure and developing resources while maintaining communication with the original site and overseeing transitional processes, we have come across unforeseen limitations. Fortunately, these hurdles have provided opportunities to learn, grow, and develop new processes to benefit the future of the study. While it has been an arduous process, we have learned that maintaining close and open communication among all parties is critical to a successful grant transition.

\section{Recommendations}

Primarily, communication is essential. As soon as it is known that a PI will be requesting to transfer a grant, it is imperative that he/she has a discussion with the current institution's dean or associate dean for research and with the counterpart at the receiving institution. Being proactive about and maintaining quality communication is necessary for both institutions. Concurrently, the PI can be informing the study program officer of the impending transfer and seek guidance as to NIH process requirements. Understanding the NIH guidelines up front will help for a smoother transition. Next, optimal timing for relinquishment and acceptance can be determined such that budgetary modifications can be negotiated and approved with minimal loss of time for study activities. These timelines will dictate when it is appropriate to ship materials to the new site as well as data and systems transfers. The goal of a grant transfer is to have everything in place at the new institution in order to start study activities as soon as the transfer is official. 
In addition to starting procedures and data collection at the new site, it is just as important to accommodate completion of study participants at the original site with smooth closeout operating procedures.

\section{References}

1. Cooperberg M, Grossfield G, Lubeck D, Carroll P. National practice patterns and time trends in androgen ablation for localized prostate cancer. Journal of the National Cancer Institute. 2003; 95: 981-9

2. Higano C. Side effect of androgen deprivation therapy: Monitoring and minimizing toxicity. Urology. 2003; 61 (2 suppl 1): 328

3. Johansson E, Bill Axelson A, Holmberg L, Onelov E, Johansson JE, Steineck G, et al. Time, symptom burden, androgen deprivation, and self-assessed quality of life after radical prostatectomy or watchful waiting: the Randomized Scandinavian Prostate Cancer Group Study Number 4 (SPCG04) clinical trial. Eur Urol. 2009; 55 (2): 422-30. Epub 2008/09/12. Doi: 10.1016/j.eururo.2008.08.054. PubMed PMID: 1873877. NIHMS\#: NIHMS88484.

4. Kattan MW. Measuring hot flashes in men treated with hormone ablation therapy: an unmet need. Urol Nurs. 2006;26(1):13-8. Epub 2006/03/28. PubMed PMID: 16562382.
5. Labrie F, Cusan L, Gomez J, Belanger A, Candas B. Long-term combined androgen blockade along for localized prostate cancer. Molecular Urology. 1999;3:217-26.

6. Levine G, D'Amico A, Berger P, Clark P, Eckel R, Keating N, et al. Androgen-Deprivation Therapy in Prostate Cancer and Cardiovascular Risk. CA: A Journal for Clinicians. 2010;60(3):294-01. PubMed Central PMCID: PMC3049943.

7. Kabir S, Mancuso P, Rashid P. Androgen deprivation therapy-managing side effects. Aust Fam Physician. 2008;37(8):641-6. Epub 2008/08/16. PubMed PMID: 18704213.

8. Nishiyama T, Ishizaki F, Anraku $\mathrm{T}$, Shimura H, Takahashi K. The influence of androgen deprivation therapy on metabolism in patients with prostate cancer. Journal of Clinical Endocrinology and Metabolism. 2005;90:657-66.

9. Saylor PJ, Smith MR. Metabolic complications of androgen deprivation therapy for prostate cancer. J Urol. 2013;189(1 Suppl):S3442; discussion S3-4. Epub 2012/12/19. doi: 10.1016/j.juro.2012.11.017. PubMed PMID: 23234628.

10. Tsai H, D'Amico A, Sadetsky N, Chen M, Carroll P. Androgen deprivation therapy for localized prostate cancer and the risk of cardiovascular mortality. Journal 
of the National Cancer Institute. 2007;99(20):1515-24.

11. Basaria S, Muller D, Carducci M, Egan J, Dobs A. Hyperglycemia and insulin resistance in men with prostate carcinoma who receive androgen deprivation therapy. Cancer. 2006;106:581-8.

12. Lubeck D, Grossfeld G, Peter R. The effect of androgen deprivation therapy on health-related quality of life in men with prostate cancer. Urology. 2001;58(suppl. 2A):94-100.

13. Efstathiou JA, Bae K, Shipley WU, Hanks GE, Pilepich MV, Sandler HM, et al. Cardiovascular mortality after androgen deprivation therapy for locally advanced prostate cancer: RTOG 8531. J Clin Oncol. 2009;27(1):92-9. Epub 2008/12/03. doi: 10.1200/jco.2007.12.3752. PubMed PMID: 19047297; PubMed Central PMCID: PMC2645096.

14. Saigal C, Gore J, Krupski T, Hanley J, Schonlau M, Litwin M. Androgen deprivation therapy increases cardiovascular morbidity in men with prostate cancer. Cancer. 2007;110:1493-500.

15. Penson D. ADT Risk. In: Maliski SL, editor. Thousand Oaks2010.

16. Klein JB, Nguyen CT, Saffore L, Modlin C, 3rd, Modlin CS, Jr. Racial disparities in urologic health care. J Natl Med Assoc. 2010;102(2):108-17. Epub 2010/03/03. PubMed PMID: 20191923.
17. ACS. Cancer Facts and Figures. American Cancer Society, 2012.

18. Bennett C, Ferreira M, Davis T, Kaplan J, Weinberger M, Kuzel T, et al. Relation between literacy, race, and stage of presentation among low-income patients with prostate cancer. Journal of Clinical Oncology. 1998;16(9):3101-4.

19. Bradley C, Given C, Roberts C. Late stage cancers in a Medicaidinsured population. Medical Care. 2003;41(6):722-8.

20. Danley KL, Richardson JL, Bernstein L, Langholz B, Ross RK. Prostate cancer: trends in mortality and stage-specific incidence rates by racial/ethnic group in Los Angeles County, California (United States). Cancer Causes Control. 1995;6(6):492-8. PubMed PMID: 8580296.

21. Dayal HH, Chiu C. Factors associated with racial differences in survival for prostatic carcinoma. J Chronic Dis. 1982;35(7):553-60.

22. Eisen $S$, Waterman B, Skinner C, Scherrer J, Romeis J, Bucholz K, et al. Sociodemographic and health status characteristics associated with prostate cancer screening in a national cohort of middle-aged male veterans. Urology. 1999;53:516-22.

23. Halpern M, Ward E, Pavluck A, Schrag N, Bian J, Chen A. Association of insurance status and ethnicity with cancer stage at diag- 
nosis for 12 cancer sites: A retrospective analysis. Lancet.

2008;9:222-31. PMID: 18282806.

24. Gilligan T. Social disparities and prostate cancer: Mapping the gaps in our knowledge. Cancer Causes and Control. 2005;16:4553.

25. Penson D, Stoddard M, Pasta D, Lubeck D, Flanders S, Litwin MS. The association between socioeconomic status, health insurance coverage, and quality of life in men with prostate cancer. J Clin Epidiol. 2001;54.

26. Allicock M, Campbell MK, Valle CG, Barlow JN, Carr C, Meier A, et al. Evaluating the implementation of peer counseling in a church-based dietary intervention for African Americans. Patient Educ Couns. 2010;81(1):3742. Epub 2010/01/02. doi: 10.1016/j.pec.2009.11.018. PubMed PMID: 20044231.

27. Enger $S$, Van Den Eeden $S$, Sternfeld B, Loo K, Quesenberry Jr C, Rowell S, et al. California men's health study (CMHS): A multiethnic cohort in a managed care setting. BMC Public Health. 2006;6(172-181).

28. Haskell W, Lee I, Pate R, Powell $\mathrm{K}$, Blair S, Franklin B, et al. Physical activity and public health: Updated recommendations for adults from the American College of Sports Medicine and American Heart Association. Circulation. 2007;116:1081-93.
29. Ervin RB. Prevalence of metabolic syndrome among adults 20 years of age and over, by sex, age, race and ethnicity, and body mass index: United States, 2003-2006. Natl Health Stat Report. 2009(13):1-7. Epub 2009/07/29. PubMed PMID: 19634296.

30. Martinez SM, Ainsworth BE, Elder JP. A review of physical activity measures used among US Latinos: guidelines for developing culturally appropriate measures. Ann Behav Med. 2008;36(2):195207. Epub 2008/10/16. doi: 10.1007/s12160-008-9063-6. PubMed PMID: 18855091.

31. Ramirez A, Chalela P, Gallion K, Velez L. Energy balance feasibility study for Latinas in Texas: A qualitative assessment. Prev Chronic Dis. 2007;4:A98.

32. Sobalske M. Health care seeking among Mexican American men. J Trans Cult Nurs. 2006;17(2):12938.

33. Thompson E, Berry D, Nasir L. Weight management in AfricanAmericans using church-based community interventions to prevent type 2 diabetes and cardiovascular disease. J Natl Black Nurses Assoc. 2009;20(1):59-65. Epub 2009/08/21. PubMed PMID: 19691185.

34. Two Feathers J, Kieffer E, Palmissano G, Anderson M, Sinco B, Janz N, et al. Racial and ethnic approaches to community health (REACH) Detroit partnership: 
Improving diabetes-related outcomes among African American and Latino adults. Am J Public Health. 2005;95(9):1552-60.

35. Andersen R, Lewis S, Giachello A, Aday L, Chiu G. Access to medical care among Hispanic population of the southwestern United States. J Health Soc Behav. 1981;22(1):78-89.

36. Association AH. Heart Facts 2007:

Latino/Hispanic Americans. American Health Association, 2007.

37. Colleran K, Richards A, Shafer K. Disparities in cardiovascular disease risk and treatment: Demographic comparison. J Investig Med. 2007;55(8):415-22.

38. Meadows TA, Bhatt DL, Hirsch AT, Creager MA, Califf RM, Ohman EM, et al. Ethnic differences in the prevalence and treatment of cardiovascular risk factors in US outpatients with peripheral arterial disease: insights from the reduction of atherothrombosis for continued health $(\mathrm{REACH})$ registry. Am Heart J. 2009;158(6):1038-45. Epub 2009/12/05. doi:

10.1016/j.ahj.2009.09.014. PubMed PMID: 19958873.

39. Valdez R, Giachello A, Rodriguez-Trias H, Gomez P, de la Rocha $\mathrm{C}$. Improving access to health care in Latino communities. Public Health Report. 1993;108(5):5349.
40. Vega WA, Rodriguez MA, Gruskin E. Health disparities in the Latino population. Epidemiol Rev. 2009;31:99-112. Epub 2009/08/29. doi: 10.1093/epirev/mxp008. PubMed PMID: 19713270.

41. Zeno SA, Kim-Dorner SJ, Deuster PA, Davis JL, Remaley AT, Poth M. Cardiovascular fitness and risk factors of healthy African Americans and Caucasians. J Natl Med Assoc. 2010;102(1):28-35. Epub 2010/02/18. PubMed PMID: 20158133.

42. Krupski T, Fink A, Kwan L, Maliski S, Connor S, Clerkin B, et al. Health-related quality of life in low- income, uninsured men with prostate cancer. Journal of Health Care for the Poor and Underserved. 2005;16(2):375-90.

43. Zavala MW, Maliski SL, Kwan L, Miller DC, Fink A, Litwin MS. Longitudinal quality of life in low-income men in a statefunded prostate cancer treatment program. J Health Care Poor Underserved. 2008;19(1):200- 14. Epub 2008/02/12. doi: 10.1353/hpu.2008.0026. PubMed PMID: 18263996.

44. Maliski SL, Rivera S, Connor S, Lopez G, Litwin MS. Renegotiating masculine identity after prostate cancer treatment. Qual Health Res. 2008;18(12):1609-20. Epub 2008/10/29. doi: 10.1177/1049732308326813. PubMed PMID: 18955461. 